CARNETS DE Carnets de géographes

GÉOGRAPHES.

$7 \mid 2014$

Les espaces de l'entre-deux

\title{
L'entre-deux comme résilience. Les relocalisations sur le volcan merapi (Java)
}

Jean-Baptiste Bing

\section{(2) OpenEdition}

Journals

Édition électronique

URL : http://journals.openedition.org/cdg/457

DOI : $10.4000 /$ cdg.457

ISSN : 2107-7266

Éditeur

UMR 245 - CESSMA

Référence électronique

Jean-Baptiste Bing, "L'entre-deux comme résilience. Les relocalisations sur le volcan merapi (Java)», Carnets de géographes [En ligne], 7 | 2014, mis en ligne le 01 décembre 2014, consulté le 19 avril 2019 URL : http://journals.openedition.org/cdg/457 ; DOI : 10.4000/cdg.457

La revue Carnets de géographes est mise à disposition selon les termes de la Licence Creative Commons Attribution - Pas d'Utilisation Commerciale - Pas de Modification 4.0 International. 


\title{
L'ENTRE-DEUX COMME RÉSILIENCE. LES RELOCALISATIONS SUR LE VOLCAN MERAPI (JAVA)
}

\author{
JEAN-BAPTISTE BING \\ Université de Genève \\ Faculté des sciences de la société \\ Département de géographie et environnement \\ jean-baptiste.bing@unige.ch
}

\section{Résumé}

Suite à la destruction de leurs villages durant l'éruption d'octobre-novembre 2010, plusieurs communautés villageoises du flanc Sud du Merapi ont été relocalisées hors de la zone de danger principale. Pensées par les autorités en collaboration avec les représentants des habitants, ces opérations ont posé et posent de délicats problèmes socioculturels, touchant tant au quotidien qu'aux relations complexes de la population avec sa montagne, et ce dans un contexte où la modernité engendre sur le temps long une double mutation, économique et métaphysique. Cet article, écrit à la demande de représentants de l'un des "forums hybrides " locaux, propose d'examiner la situation des villages relocalisés en les pensant comme espaces de l'entre-deux afin de relier enjeux de représentation, de territorialité et d'économie à court et moyen terme. II conclut à une nécessaire interdisciplinarité tant dans l'analyse que dans l'action, organisée au plus près des habitants et en coordination avec eux, afin de répondre à la multiplicité des enjeux aux différentes échelles, spatiales comme temporelles, où ils s'expriment.

\begin{abstract}
After their villages were destroyed by the eruption of October and November 2010, several village communities of the Mount Merapi's southern flank were relocated outside the main hazard zone. Elaborated by authorities in collaboration with representatives of the inhabitants, these operations caused and cause delicate socio-cultural problems about everyday life as about the complex relationships between population and its mountain, against a background of a long term mutation in economics and metaphysics linked to modernity. Written at the request of representatives from a local "hybrid forum", this article proposes to examine the situation of relocated villages, seeing them as between-spaces in order to link issues of representation, of territoriality and of economics at short and middle term. This article concludes with the necessity of interdisciplinarity for analysis as for action, organized as closely as possible to the inhabitants and coordinated with them, in order to face the multiplicity of challenges at several spatial and temporal scales.
\end{abstract}




\section{Introduction}

26 octobre 2010 : suite à un gros mois de réveil progressif, le Merapi (Java, Indonésie) entre dans une phase éruptive plus de quatre ans après la précédente. Dès le 5 novembre, le bilan est lourd: plus de 300 morts, plusieurs villages ravagés. Depuis des décennies, jamais le volcan n'avait autant tué et détruit. Pendant que les volcanologues s'interrogeaient sur un possible changement de régime éruptif, les habitants frappés par le désastre déménageaient de centre d'hébergement en centre d'hébergement (Melchior 2011). Deux ans plus tard, certaines "communautés villageoises" $\left(\mathrm{marga}^{1}\right)$ ont regagné leur village et rebâti sur les ruines; d'autres ont accepté l'offre gouvernementale de "relocalisation " (relokasi ${ }^{2}$ ) dans des villages neufs bâtis quelques kilomètres plus bas (cf. figure 1), sur des terres en friche achetées à des propriétaires privés et situées en marges d'autres villages ${ }^{3}$.

En janvier-février 2013, un séjour de recherche sur le Merapi a permis de rencontrer des acteurs impliqués dans ces opérations : habitants, duko, " médiateurs " (cf. ci-dessous " Les médiateurs, interlocuteurs privilégiés »), techniciens. Illustration de la sérendipité, ce séjour effectué dans le cadre de ma thèse devait être consacré aux questions forestières. La relocalisation ayant affecté le fonctionnement de l'économie des marga concernées et donc le rapport des habitants à la forêt sommitale, je fus amené à me pencher aussi sur la relocalisation elle-même. Souhaitant que les habitants du Merapi (souvent interrogés par des scientifiques - cf. ci-dessous "Décentrer le regard » - mais recevant peu de retours) bénéficient des travaux effectués chez eux, les responsables du forum al-Qodir ${ }^{4} \mathrm{~m}^{\prime}$ ont demandé ce que la "théorie géographique " (teori ilmu geografi) pourrait apporter quant aux difficultés posées par la relokasi.

Cet article aborde ainsi les problèmes rencontrés dans la concrétude du quotidien (voisinage, ressources, ...) et les lie à des enjeux plus vastes, moins immédiats mais non moins réels (prévention des risques, modernisation de la société....). II s'agit d'envisager comment intégrer des phénomènes d'ordres très variés dans une stratégie globale visant à pallier aux carences des politiques publiques. Considérer ces enjeux en pensant les villages relocalisés comme espaces de l'entre-deux semble heuristiquement porteur : après un point méthodologique, on définira les acteurs porteurs de dynamiques dans cet entre-deux, avant d'examiner les changements de territorialités liés à la nouveauté de ces espaces et de se pencher sur les questions économiques qui en découlent.

\footnotetext{
${ }^{1}$ Les termes indonésiens en italique revinrent régulièrement chez les personnes interrogées.

${ }^{2}$ Ces communautés seront donc désignées dans la suite du texte par les expressions " marga relocalisées", " villages relocalisés » ou " communautés relocalisées ".

3 Une " commune » (desa), dirigée par un «chef de commune » (kepala desa), regroupe administrativement plusieurs « villages » (dusun), dirigés chacun par un duko.

${ }^{4}$ "Forum hybride » rassemblant les duko, kepala desa, techniciens des administrations et médiateurs. J'en profite pour remercier ceux qui m'ont accueilli et guidé sur place, notamment pak Wiranata et mas Asep.
} 
Figure 1 : Carte de situation du Merapi et des villages ${ }^{5}$ cités

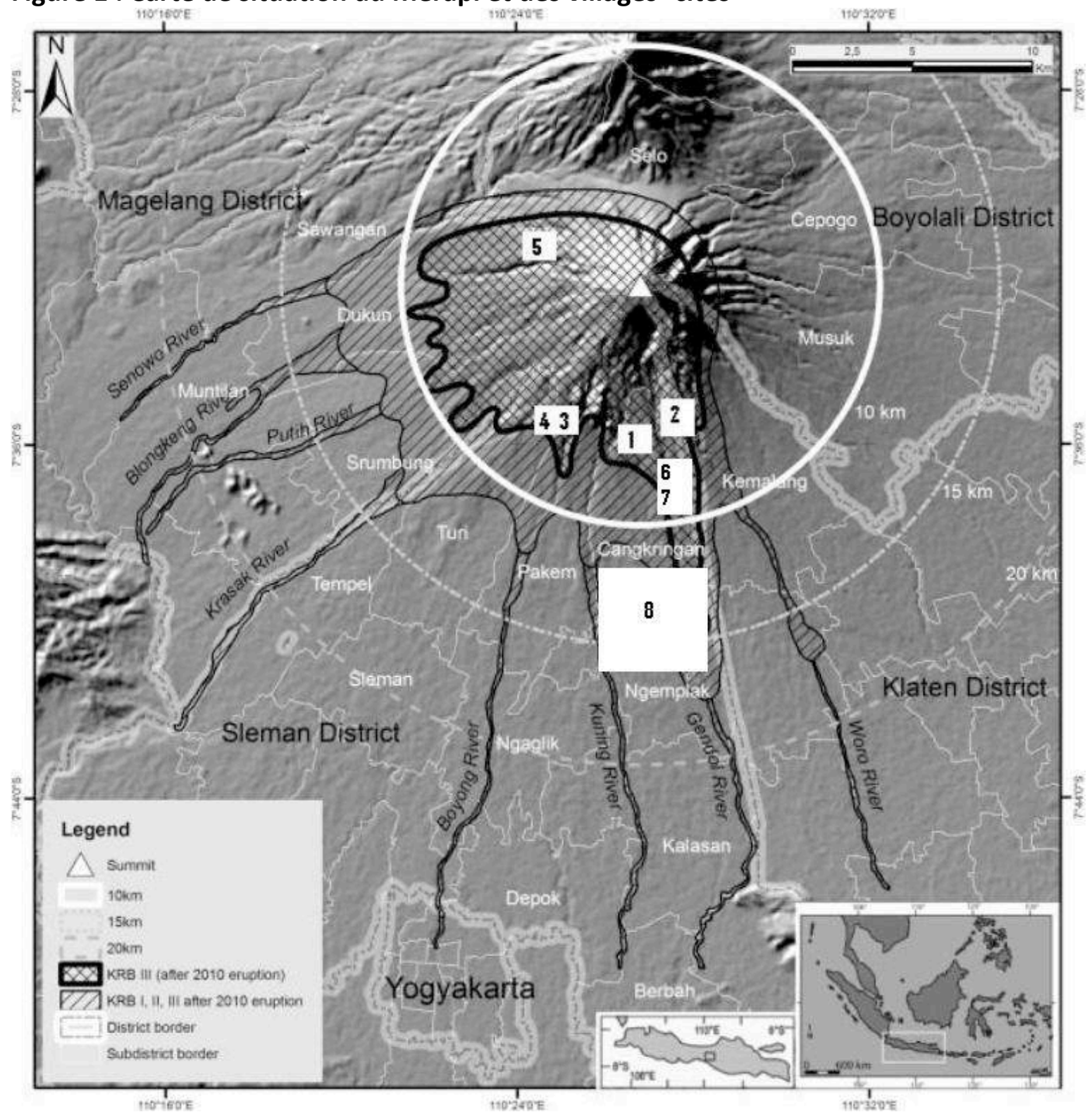

1 : Kinahrejo ; 2 : Kalitengah Lor ; 3 : Turgo ; 4 : Tritis ; 5 : Babadan ; 6 : Kaliadem ; 7 : Jambu ; 8 : Zone des communes accueillant les relocalisations et de Pentingsari (Sud-ouest). Fond de carte tiré de Mei, Lavigne et al. 2013.

\section{Comment appréhender l'entre-deux ?}

En raison des complexités d'une situation par essence provisoire, fluide et en évolution rapide, il est impossible d'espérer tout voir ou écouter tout le monde. En amont du séjour puis sur place, le choix a été fait de se limiter à des données qualitatives (observations, entretiens,...) recueillis sur certains lieux précis, sans s'interdire pour autant ni des détours

\footnotetext{
${ }^{5}$ Dans le cas de Kinahrejo (1), Kaliadem (6) et Jambu (7), il s'agit du site historique des villages: la terre appartient toujours aux villageois et ceux-ci viennent y travailler (cf. ci-dessous «Quel impératif communautaire pour l'économie ? ») ; mais la relocalisation a transféré les zones d'habitat en (8).
} 
par d'autres endroits (site de surveillance de Babadan) ni des recoupements avec d'autres types de données.

\section{Comparer les lieux}

Pour des raisons pratiques (temps disponible, distances aux villages et précédent séjour en 2009), l'étude s'est focalisée sur quatre marga: celles de Kinahrejo, Kaliadem, Jambu et Kalitengah Lor. Situés à la lisière de la forêt qui borde le sommet, les quatre villages d'origine se trouvaient dans la zone décrétée inconstructible par les autorités, mais où les implantations restaient tolérées en raison de leur ancienneté. Seuls quelques kilomètres séparaient les villages, et leurs marga avaient (et conservent) une structure sociale et culturelle semblable; mais la relocalisation a engendré de brutales mutations des conditions de vie qui, désormais, diffèrent fort d'une marga relocalisée à l'autre (les autorités procèdent à la relocalisation marga par marga, sans les mélanger, afin de laisser le plus possible intactes les structures sociales). Celles de Kinahrejo, Kaliadem et Jambu ont accepté la relocalisation, et ont ainsi eu droit aux aides gouvernementales. Dans les faits seul Kinahrejo baru ( Kanahrejo neuf »), qui a bénéficié d'une forte mobilisation privée et associative, a été achevé et mis à la disposition de la communauté en juin 2012 ; pour les marga de Kaliadem et de Jambu, le processus (dépendant surtout de l'aide publique) prit bien plus de temps. Quant aux habitants de Kalitengah Lor, qui ont " choisi l'indépendance » (pilih mandiri) en rebâtissant sur place, ils ont rapidement retrouvé leur vie d'avant. Une démarche comparative s'impose ainsi au sein du terrain étudié.

En outre, deux types d'apports extérieurs permettront d'éclairer les différentes dimensions des dynamiques sociales en cours: d'une part, tirer des enseignements d'expériences semblables s'étant déroulées ailleurs et, d'autre part, interroger des disciplines a priori éloignées. À ce stade de la réflexion, un risque apparaît : celui de la confusion. Pour y parer, une précaution méthodologique s'impose: si l'on ne s'interdit a priori aucune piste de réflexion transversale, les particularités de chaque cas interdisent toute généralisation, bien que l'on puisse considérer le flanc Sud du Merapi comme un tout vis-à-vis de l'extérieur. II convient de distinguer chaque marga et chaque village -et de procéder à une comparaison dans l'analyse et à une adaptation dans les propositions (Callon et al. 2001 : 14), et non d'imiter ce qui a été fait ailleurs.

\section{Décentrer le regard}

Seule une minorité des (nombreux) chercheurs étrangers qui travaillent sur le Merapi œuvre dans les humanités, et peu se privent de soutien officiel en venant sur place sans être chapeautés par une institution locale. Pour des raisons de nature de visa (tourisme et non recherche), je dus m'en contenter; occuper ainsi une niche spécifique par rapport aux autres scientifiques me permit de me tenir hors des jeux de pouvoir dans lesquels les dites institutions locales étaient engagées tout en rencontrant, de manière officieuse, des gens qui en étaient issus. Scientifique hors institution et, par ailleurs, Européen lié par des liens de famille à l'Indonésie : ce statut peu courant engendre un décentrement du regard, potentiellement fécond (Lapierre 2006).

Outre la nécessité éthique de présenter, lors des entretiens, la démarche suivie et son but, préciser cet entre-deux épistémologique facilitait souvent - pas toujours - les confidences lors de conversations informelles. Revers de la médaille: cette méthode extensive de 
cueillette de données rendait la prise de notes (peu propice à une discussion à bâtons rompus) plus hasardeuse. D'où un premier biais: pour valoriser les notes ramenées, rédigées en un mélange de français et d'indonésien et mêlant observations et comptesrendus d'entretiens, il a fallu les confronter à d'autres données issues de la littérature grise émise par les institutions locales et à la vaste littérature déjà écrite au sujet du Merapi ${ }^{6}$.

Parler la langue nationale permet d'évoluer sur place de manière autonome. Ainsi, les entretiens se déroulaient en indonésien, langue maîtrisée par la quasi-totalité de la population du volcan (sauf parmi les plus de 70 ans, où la proportion de javanophones exclusifs est plus élevée). Toutefois, mes interlocuteurs (parlant pour la plupart javanais comme langue première) et moi-même (francophone) communiquions via une langue seconde : l'expression et la réception passaient ainsi - second biais - par un filtre qui voile en partie le sens, et rend moins subtil le jeu possible des nuances; filtre d'autant moins négligeable que les mots employés ne s'inscrivaient pas dans les mêmes pratiques du monde et n'avaient ainsi ni le même sens ni la même valeur. Un exemple: le mot kebun ( " plantation ») ne désignait pour moi qu'un concept et un paysage singulièrement pauvres ; pour eux, il est - entre autres - un lieu du quotidien, bâti dans le passé et garantie pour l'avenir, un espace agricole face à la forêt (hutan) et à la friche (ladang)...

Ce choix de croiser les points de vue (y compris celui du chercheur) doit donc permettre de dégager des pistes de réflexion visant, par-delà les biais cités, à appuyer les projets de relocalisation en cours en intégrant dans l'analyse un maximum de paramètres dont l'exploitation concrète pourraient s'appuyer les uns sur les autres et non se contredire.

\section{Qui pour dépasser l'incommensurabilité ?}

Un tel objectif impose de tenir compte des conflits qui existent au sujet du volcan, de son usage et de l'interprétation de ses manifestations (Bing 2009), non pour les supprimer mais parce qu'il y a là une condition minimale d'une collaboration entre les partis en présence.

\section{Les médiateurs, interlocuteurs privilégiés}

Berliner (2010: 14) définit les " médiateurs " comme une série d'acteurs, humains ou nonhumains, individuels ou collectifs, intervenant dans le "faire-passer ». Ici, seront distingués les acteurs humains (" médiateurs", en un sens restreint) des supports non-humains ( médias ") de ce processus. Sont "médiateurs» des individus qui, disposant d'une légitimité reconnue par tous les groupes en présence, servent d'intermédiaires, favorisent la communication et l'établissement du consensus. Pour cela, ils élaborent et manient les " médias " qui incarnent l'échange et la co-construction de nouveaux savoirs et savoir-faire entre les acteurs (tels les experts et les citoyens, Callon et al. $2001: 20-21$ ) dont les modes de pensée et d'action présentent un certain degré d'incommensurabilité.

L'ouverture ancienne du Merapi sur l'extérieur et le faible enclavement des villages (surtout sur le flanc Sud), multiplient les potentiels médiateurs. Trois types se dégagent. 1/ Des personnes nées étrangères aux marga mais installées ou actives sur place qui, par

\footnotetext{
${ }^{6}$ Ne sont précisés ici que les principaux renvois. Pour plus de détails, cf. Bing (2009) et Bing (à paraître).
} 
I'ancienneté de leur présence et/ou leur investissement social et/ou leurs compétences ont gagné la confiance de tous. 2/ Des personnes nées sur place, qui ont acquis une autorité et/ou un savoir et/ou un savoir-faire particuliers. 3/ Moins en vue mais pas moins essentiels, des habitants qui travaillent pour une institution officielle implantée sur le Merapi : Taman Nasional Gunung Merapi (Parc national du Merapi), Kraton (palais du sultan-gouverneur de la région), Badan Penanggulangan Bencana Daerah et Rekompak (administrations locales de prévention des risques et de la reconstruction), etc.

Ce rôle central des médiateurs pose deux questions. D'abord celle de leur représentativité et de leur mode de sélection (Callon et al. 2001). Ensuite, celle du consensus : en pays javanais, en effet, le positionnement réciproque de chaque acteur est strictement défini en raison de l'importance de la " face » et de la hiérarchie (Bing 2014). Obtenir l'accord de la collectivité concernée par un projet est fondamental pour le développer à long terme. En témoignent $a$ contrario le fiasco de la prévention des risques en 2006 : quand les " gens d'ici » (wong sini, habitants du Merapi) font bloc contre les "gens de là-bas " (wong sono, scientifiques et administratifs venus de Yogyakarta ou d'ailleurs), alors rien ne peut avoir lieu (Bing 2009). Cela suppose certes un temps long de socialisation (sosialisasi ${ }^{7}$ ), mais la réussite des projets en dépend. Mais ce consensualisme porte bien des ambigüités. D'abord, un consensus apparent peut masquer des non-dits facteurs de conflits futurs - ce qui n'est pas univoque non plus, puisqu'une dynamique lancée peut contrebalancer les conflits potentiels. Mais surtout, "le consensus est souvent le masque qui cache les rapports de domination et d'exclusion. [...] La politique est l'art de traiter les désaccords, les conflits, les oppositions, et pourquoi pas de les faire surgir, de les favoriser, de les multiplier, car c'est ainsi que des chemins inattendus s'ouvrent, que les possibles se multiplient. " (Callon et al. 2001 : 16).

\section{Les forums hybrides, lieux de concertation}

Pour être efficaces en termes de prévention comme de résilience, l'action et l'analyse doivent parvenir à connecter l'urgence et le long terme, le quotidien aux rythmes longs, les échelles domestique et villageoise voire régionale et nationale (Dauphiné 2005). Les éruptions du Merapi ayant lieu tous les quatre à cinq ans et la densité de population dépassant les 1000 hab. $/ \mathrm{km}^{2}$ (Lavigne 2001), les enjeux socio-économiques et l'inertie sociale sont tels que toute mesure se doit d'être pérenne. L'efficacité réclame donc de l'analyse qu'elle tienne compte de la spécificité de chaque cas, et de l'action qu'elle colle aux dynamiques sociales. Ainsi, toute solution doit se voir élaborée, toute piste explorée et toute décision prise, en concertation avec les habitants concernés (Callon et al. 2001).

Que ce fût lors de réunions formelles et collectives ou d'entretiens individuels et informels, les interlocuteurs ont évoqué divers paramètres jugés déterminants pour le succès des opérations en cours. Les relocalisés doivent ainsi: s'approprier leur nouveau village, maintenir soudées leurs communautés, s'insérer dans les communes d'accueil, redévelopper des activités économiques viables sur les terres des anciens villages. Ces enjeux se résument donc à participer activement à une sortie de la condition passive de réfugié victime de catastrophe (Vásquez Lezama 2009) autrement que par un retour (illusoire et/ou impossible,

\footnotetext{
${ }^{7}$ En théorie, la sosialisasi est un temps d'échange, où chacun définit sa position dans la relation réciproque en construction. Dans les faits, le terme est souvent employé pour donner une connotation participative à un processus top-down de "vulgarisation" (dite également penyuluhan, "éclaircissement ») auprès des habitants.
} 
en tout cas indésirable) au statu quo ante. Pour illustrer cela, ils citaient en contre-exemple le cas des villages de Turgo et Tritis détruits en 1994 : malgré plus de soixante tués, un projet de relocalisation incomplet et mis en œuvre de façon autoritaire n'a finalement abouti qu'à la relocalisation de quelques familles.

D'où l'importance de créer des "forums hybrides " (Callon et al. 2001), instances hors des cadres officiels où peuvent se négocier des collaborations (kerja sama) et s'échanger des expériences (tukar pengalaman). Après le fiasco de l'évacuation de 2006, le "forum Merapi " devait rapprocher scientifiques, administratifs et villageois; dès 2008, témoignaient de son succès la fin des vols de matériel et l'implication de médiateurs de types 2 et 3 dans la surveillance du volcan (Bing 2009). Dans le cas ici étudié, la pesantren alQodir $^{8}$, sise au pied de la zone de relocalisation, a d'abord joué un rôle-clé dans l'accueil des réfugiés et l'organisation de l'action sociale en octobre-novembre 2010; depuis, s'y déroulent des réunions régulières entre les chefs des villages relocalisés, les chefs des communes et des villages d'accueil, des agents de la Rekompak, des médiateurs et des journalistes. Le volontarisme de son charismatique kyai (médiateur de type 2) joua un grand rôle dans ce processus.

Dans une situation sociale tendue, ce sont donc des individus (les médiateurs) capables de naviguer entre les partis et des institutions (les forums hybrides) formées hors des cadres habituels qui peuvent fluidifier les relations et engendrer une dynamique de concertation. Situés dans un entre-deux métaphoriques, ils doivent donc permettre d'établir in fine une nouvelle normalité à la place de l'entre-deux.

\section{Quelle territorialité pour les villages neufs?}

L'entre-deux des villages neufs s'exprime par leur ambigüité : situés dans des périphéries de communes, ils deviennent des centres sans que cette centralité soit identique à celle des villages détruits. Or, plus de trois ans après l'éruption de 2010, et alors qu'une faible éruption a déjà en lieu en novembre 2013, analyser les premiers mois passés par les habitants de Kinahrejo dans leur nouvel environnement éclaire les tendances semblables se font jour dans les autres marga relocalisées.

\section{Retrouver une topophilie symbiotique}

Raffestin (1986) définit la territorialité comme la relation à l'altérité humaine et à l'extériorité physique, débouchant sur une sémiotisation d'un lieu qui, ainsi, fait sens pour ses habitants. Quant à la métaphore de la "symbiose " entre un volcan et ses habitants (Augendre 2004), elle qualifie bien la relation traditionnelle des habitants du Merapi à leur montagne; mais dans le cas des marga relocalisées, cette symbiose a été cassée. La retrouver serait à la fois " empreinte et matrice " ${ }^{9} d^{\prime}$ une relocalisation réussie. Cela suppose que les habitants développent un lien topophilique aux nouveaux villages, transformant ces « espaces » en « lieux » (Tuan 2006) via le passage par l'entre-deux.

\footnotetext{
${ }^{8}$ Pesantren : école coranique, dirigé par un kyai (dignitaire religieux traditionnel; selon Hiroko Horikoshi dans Kyai dan perubahan sosial, les kyai constituent l'un des moteurs des changements sociaux à Java depuis l'époque coloniale ; en retrouver parmi les médiateurs n'est donc pas surprenant).

${ }^{9}$ Expression par laquelle A. Berque désigne le paysage, "empreinte et matrice » des sociétés; l'expression est donc ici quelque peu détournée, quoique fidèle à son sens premier.
} 
Territorialité, symbiose et topophilie se construisent au quotidien, par des habitudes peu à peu acquises, mais aussi quand un moment fort marque un endroit du sceau du souvenir et le transforme en haut-lieu (Pitte 2010). Un évènement au carrefour de ces deux temporalités est la célébration officielle d'inauguration du village neuf : elle signe la fin du processus d'installation, et marque le début de la phase (censée être définitive) d'habitation. Pour Kinahrejo baru, la célébration a eu lieu en juin 2012, quand bien même certains bâtiments restaient à construire. Cependant, un attribut essentiel manque à ce proto-lieu : le toponyme. Les habitants emploient soit des paraphrases comme "desa baru " (village neuf) soit le nom de l'ancien village accolé à l'épithète baru. Certes, "Kinahrejo Baru " pourrait constituer un véritable nom, à condition que cette dignité lui fût accordée par ses usagers; or pour l'instant, il ne s'agit aux dires des interlocuteurs que d'une simple expression. Ce manque d'appropriation va de pair avec une nostalgie pour l'ancien village, paré de toutes les vertus Typique de l'exil (Lapierre 2006), cette nostalgie touche notamment les plus âgés dont beaucoup espèrent à terme "habiter à nouveau là-haut " (tinggal di atas lagi) - ce qui signifierait (comme pour Turgo et Tritis) l'échec du projet de relocalisation.

Le lieu d'implantation pose enfin le défi de l'intégration des marga relocalisées au sein des communautés sur le territoire desquelles ont été implantés les villages neufs : tantôt les pratiques agronomiques diffèrent, et la venue de gros bétail pose des problèmes aux habitants déjà installés sur place; d'autres fois, se font jour des rivalités d'usages liés aux terrains occupés par les villages neufs; enfin, il peut y avoir de la jalousie à voir de nouveaux arrivants bénéficier de tout le confort moderne, quand les anciens doivent se contenter de conditions plus spartiates. Ce phénomène n'est pas non plus sans rapport avec certaines difficultés vécues dans des villages de transmigrants ${ }^{10}$ pour établir un nouveau vivreensemble (Levang 1997). Le centre d'étude du ministère de la Transmigration sis à Pentingsari (proche de Kinahrejo baru) pourrait fournir des témoignages à exploiter - et ainsi s'insérer dans un tissu rural où il apparaît comme une excroissance de l'administration.

\section{Inventer une nouvelle rurbanité}

L'ancien village de Kinahrejo faisait une quarantaine d'hectares; le nouveau en fait 1 à 1,5 (selon les estimations données). Le nombre de familles n'ayant pas changé, la densité de population a explosé. Finie la maison entourée d'un terrain privé clos par des haies vives ; place aux maisons plus petites collées les unes aux autres. L'équation est la même pour les nouveaux Kaliadem et Jambu. Dans les anciens villages, chaque famille disposait d'une réelle intimité; ce n'est plus le cas, et trouver un nouvel équilibre demande énormément d'habileté. Les médiateurs en appellent à la culture javanaise du consensus (konsensus), du " haut sens social " (sosial tinggi) et du travail en commun (gotong royong), cependant ils reconnaissent que changer des habitudes acquises au fil des décennies reste difficile. Or c'est au quotidien que ce vivre-ensemble se noue : chaque fois que l'on allume la radio un peu fort ou que l'on cuisine quelque chose d'odorant. Et derrière un sourire purement formel peut se cacher un ressentiment qui, fait de petites frustrations accumulées, finit parfois par exploser. L'enjeu représenté par ces petits détails de la vie courante est

\footnotetext{
${ }^{10}$ Le programme de transmigration organise l'envoi de paysans des "îles intérieures» (Java, Bali, Madura) dites surpeuplées, vers les « îles extérieures » (Sumatra, Bornéo, Papouasie, Moluques,...) dites sous-peuplées.
} 
considérable pour les marga et pour le programme de relocalisation : il y va de l'unité des premières et de la pérennité du second.

Par ailleurs, chaque famille disposait dans les anciens villages de suffisamment de terrain pour y construire un ou des enclos hébergeant le bétail et la volaille. À Kinahrejo baru, les rues sont dédiées uniquement à l'habitat humain et, si une étable moderne a été bâtie pour les vaches laitières, il n'y a rien pour les chèvres et les volailles. Par ailleurs, l'étable se trouve légèrement en surplomb par rapport au village et s'en distingue donc nettement. Afin de faciliter l'acceptation de cette spécialisation spatiale inédite, une cuve est prévue pour stocker les excréments des vaches et en tirer du biogaz, source d'énergie peu chère, continue et renouvelable, qui assurerait un confort accru à toute la marga... si les résultats sont conformes aux attentes, et si les enjeux techniques encore non résolus (fourniture en eau pour le bétail, par exemple) le deviennent à terme.

Enfin, bien que la relocalisation interdise aux villageois d'habiter leurs anciennes parcelles, ils demeurent propriétaires des terres et peuvent les exploiter. De même, les anciens droits sur la forêt (ramassage de bois mort et de fourrage) et sur les plantations des environs n'ont pas été abolis; pour les marga de Kaliadem et Jambu, l'agriculture demeure d'ailleurs la principale ressource, le tourisme (devenu dominant à Kinahrejo - cf. ci-dessous) apportant un complément de revenus. Or les nouveaux villages, implantés hors de la zone de danger 1 , se trouvent à plusieurs centaines de mètres d'altitude sous les anciens, et à plusieurs kilomètres par la route. Les travailleurs doivent donc désormais planifier leurs déplacements pour éviter pertes de temps et efforts inutiles, alors qu'auparavant les allers-retours pouvaient se multiplier au cours d'une journée. De plus, l'absence de tout réseau de transport public rend quasiment obligatoire la possession d'au moins une moto par foyer. Cette pendularité nouvelle entre zones d'habitat (nouveaux villages) et de travail (agriculture ou tourisme) a bouleversé la territorialité des anciens villages: autrefois " quotidienne ", elle se mâtine désormais d'un caractère « d'échange » affirmé (Raffestin 1995).

La catastrophe de 2010 et la relocalisation ont donc précipité les marga touchées dans une logique territoriale moderne faite de pendularité et de rurbanité. Or, en pays javanais comme dans le reste de l'Indonésie, les agglomérations ont connu une extension spectaculaire depuis l'Indépendance; tout près, Yogyakarta a largement débordé le Ring Road et s'est étendue, notamment le long des grands axes jusqu'à Cangkringan et Pakem ${ }^{11}$. Dans ces villages devenus ville, la densification et la pendularisation frappent aussi ; si le contexte diffère, ces expériences pourraient néanmoins fournir de précieux enseignements.

\section{Quel impératif communautaire pour l'économie?}

Les médiateurs (types 1 et 2 surtout) désignent l'évolution économique comme l'un des chantiers décisifs pour la réussite de la relocalisation. Ils soulignent d'abord l'impératif à court terme de sortir de l'assistanat (Vásquez Lezama 2009). Par ailleurs ils craignent que la course aux ressources n'affecte à moyen terme les "normes sociales javanaises " (norma masyarakat Jawa), tout en voyant dans le développement une nécessité pour retenir la

\footnotetext{
${ }^{11}$ Villes situées au bas des pentes du volcan. Cangkringan est le chef-lieu du district (kecamatan) englobant les villages étudiés; à Pakem, siège de I'Université islamique où travaille Pak Asih (cf. note 10).
} 
population sur place. Le rapport complexe - voire ambigu - à la modernité que vit la société javanaise (Bing 2014) apparaît dans cette coexistence entre espoir et appréhension au sujet de ce " fait social total ${ }^{12}$ " qu'est l'économie; on se concentrera ici sur l'influence de ces perspectives concernant le sens des lieux (dans tous les sens du mot " sens » : la signification du lieu, la manière de le connaître, sa destinée,...).

\section{Une brutale accélération locale...}

La relocalisation, tout en s'insérant dans des processus régionaux et décennaux a localement bouleversé les structures économiques précédentes. Le cas de Kinahrejo est particulièrement intéressant en raison de la brutalité du processus. Lors de l'éruption de juin 2006, Mbah Marijan, le " gardien des clés " (juru kunci) du volcan ${ }^{13}$, avait refusé d’obéir à l'ordre d'évacuation du sultan; il avait ainsi accédé à une célébrité nationale, les médias s'emparant de la figure du vieux sage proche de la nature (alami), bon musulman avec qui communiquaient les esprits du volcan. L'heureux dénouement de cette éruption (aucun décès parmi les villageois) avait contribué à renforcer son aura. Tué le 26 octobre 2010 et présenté comme un martyr du devoir et de la foi, il a fait bénéficier Kinahrejo, son village, d'une rente de situation par rapport aux autres villages détruits pour développer le tourisme.

De fait, les touristes y sont nettement plus nombreux qu'ailleurs: ni l'amusante pierreextraterrestre (batu alien à $\mathrm{Jambu}^{14}$ ) ni la mystique pierre-éléphant (batu gajah à Kaliadem ${ }^{15}$, pourtant contournée d'une manière surprenante par la lave en 2006 et en 2010), ne mobilisent autant les foules. Avant 2010, l'économie de Kinahrejo était essentiellement agricole et s'insérait dans un primat de la vie collective ; depuis l'éruption, elle est devenue libérale et tertiaire : l'agriculture n'a pas vraiment redémarré (l'élevage laitier est bloqué car le bétail tué n'a pas encore été remplacé en dépit des promesses gouvernementales) et le tourisme a explosé, jouant sur le Lava tour et sur le souvenir du juru kunci décédé. Ce dark tourism rapporte beaucoup d'argent, mais pose plusieurs questions.

Celle de sa pérennité : que se passera-t-il si le Merapi passe de mode ? Selon des médiateurs de type 1 , si le tourisme a apporté des ressources utiles à la reconstruction, recréer une certaine diversité économique comme dans les autres villages détruits (à Kaliadem et Kalitengah Lor après 2010 comme à Turgo et Tritis après 1994) serait plus prudent que de tout miser sur une mono-activité. De plus, le passage de l'agriculture au tourisme a bouleversé les conventions sociales régissant le fonctionnement économique. Certes, le développement touristique s'est fait notamment sur une base coopérative, avec répartition et collectivisation d'une partie des bénéfices; mais celles-ci ne sont pas exclusives, et

\footnotetext{
${ }^{12}$ Expression de M. Mauss.

${ }^{13}$ Le juru kunci est, en principe, un habitant chargé par le sultan de diriger en son nom la cérémonie annuelle d'offrandes aux esprits du Merapi. Dans les faits, Mbah Marijan disposait d'une aura telle que beaucoup - et peut-être lui-même dans les dernières années de sa vie - le prenaient pour une sorte de chaman. Son fils et successeur, Pak Asih s'est engagé à recentrer le rôle sur la fonction de délégué du sultan.

${ }^{14}$ Vomie lors de l'éruption de 2010, elle doit ce nom - donné par par un habitant du village, puis adopté par tous - à sa forme. Cela est révélateur d'une certaine modernisation des esprits : une génération auparavant, la pierre aurait sans doute été assimilée à l'un des penunggu (esprits gardant le volcan) plutôt qu'à un ET.

${ }^{15} \mathrm{Il}$ y a plusieurs pierres en forme d'éléphant à Java et sur le Merapi ; toutes ont plus ou moins une dimension sacrée. Les éruptions de 2006 et 2010 ont renforcé la sacralité de celle de Kaliadem, la lave ayant contournée et épargnée la pierre alors qu'elle dévastait tout alentour.
} 
I'individualisme se développe. Par ailleurs, chaque foyer ayant construit son échoppe (warung) pour capter l'attention et l'argent des touristes, la concurrence se développe : à quelles conditions un fonctionnement coopératif et une vie communautaire traditionnelle restent-ils possibles dans une économie de marché ? Enfin, cette concurrence est très loin d'être "pure et parfaite ${ }^{16}$ ": selon l'endroit où se situait leur maison (près de l'entrée du village ou de la maison de Mbah Marijan), certains bénéficient d'emplacements stratégiques, donc d'une rente de situation; d'autres, ayant pu investir des sommes importantes, ont mis en place des services diversifiés et attractifs (quads, motos,...) face auxquels ceux qui n'ont qu'un peu de nourriture à proposer peuvent avoir du mal à lutter.

\section{... d'un processus plus ancien et régional}

Autrefois fondée sur les cultures vivrières et les plantations, l'économie du Merapi a vu se développer l'élevage laitier (destiné à approvisionner les grandes villes) et le tourisme (national et étranger); cette double diversification va de pair avec un désenclavement des villages qui autorise une plus grande mobilité tant physique que sociale. Ce processus comporte certes des risques, inhérents à toute économie en mutation rapide, mais il n'est pas sans avantages: l'accroissement de la population du Merapi a ainsi pu se faire tout en maintenant une population rurale importante sur place. Même les marga du haut du Merapi, peu touchées jusque là et restées relativement plus enclavées que celles du bas, cherchent à s'ouvrir et mènent une politique active de développement touristique. À Kalitengah Lor, en dépit du choix des habitants de refuser d'obéir aux consignes de relocalisation, le sultan Hamengku Buwono X a inauguré, le $1^{\text {er }}$ janvier 2013, l'infrastructure destinée à l'accueil des touristes. La participation active des marga à leur propre modernisation économique constitue un gage de responsabilité qui les place en position de force face aux desiderata des autorités.

Et cela particulièrement quand ce développement économique ne se fait pas au détriment du sens communautaire: la réussite du projet de village touristique (desa wisata) à Pentingsari doit en grande partie sa réussite à une organisation solide permettant d'intégrer un maximum de monde dans le projet et de répartir ses bénéfices entre tous. Ici, le marché est clairement mis au service de la collectivité, et tout est fait pour éviter qu'un trop grand succès ne fasse voler en éclat l'unité du village. De même, en situation de crise, la cohésion sociale passe avant tout : en février 2013, un double décès intervenu dans la nuit a obligé les responsables du village à annuler un séjour de plus de 400 personnes prévu durant plusieurs jours, afin de préparer les cérémonies funèbres : gros manque à gagner certes, mais surtout solidarité réaffirmée.

Les expériences tentées en Indonésie par les promoteurs du microcrédit (internationaux comme la banque Grameen, ou locaux comme les " coopératives de crédit " ou kopdit ${ }^{17}$ ) auraient beaucoup d'intérêt à être étudiées de près. En effet, elles combinent dimensions collective et individuelles (Yunus 2006) d'une manière qui n'est pas sans évoquer l'utilisation coopérative des fonds d'aide à Kinahrejo. Or, la diversification économique concomitante au désenclavement des villages du Merapi va de pair avec la libéralisation de l'économie. Sur le volcan, il n'y a pour l'instant pas de kopdit; mais divers micro-projets s'en rapprochent: coopératives paysannes, tontines (arisan) des groupes de femmes... À moyen terme, monter des projets plus importants (et officiels) pourrait favoriser la diversification économique déjà

\footnotetext{
${ }^{16}$ Expression des théoriciens néolibéraux.

${ }^{17}$ Acronyme de Koperasi kredit.
} 
en cours; ce pourrait aussi être une piste de réflexion pour les médiateurs de type 1 qui suggèrent de consacrer aux investissements à long terme une partie de l'aide publique reçue suite aux catastrophes, dans l'idée de démultiplier l'efficacité de cette aide.

\section{Conclusion}

L'éruption de 2010 a provoqué une rupture à plusieurs titres. Sa violence inhabituelle et ses conséquences sociales en ont fait une catastrophe au sens cindynique et systémique du terme (Péguy 2001), puisqu'elle a débouché sur une situation inédite et radicalement nouvelle, dépassant souvent (sauf pour Kalitengah Lor) les capacités de résilience habituelles des marga touchées. Tant en termes d'habitat que de perspectives économiques, des dynamiques nouvelles, bien que s'inscrivant dans des tendances longues, engendrent une instabilité sociale délicate à maîtriser. Pour cela, des moyens conséquents - scientifiques, techniques et financiers - sont mis en œuvre par les différents acteurs en présence : cela est du ressort du gouvernement provincial et des corps publics chargés de la recherche, de l'aménagement et de la relocalisation. Cependant, plusieurs témoignages laissent entendre que, dans l'urgence, c'est le soutien de la société civile qui fut déterminant et que, à moyen terme, les administrations nationales et provinciales se montrent peu efficaces.

Loin de se cantonner dans un rôle de victimes profitant de la "légitimité du malheur " (Vásquez Lezama 2009 : 196) pour s'installer dans " un ordre détaché des réalités locales » (ibid : 205), les habitants du Merapi jouent un rôle actif. Les marga prennent en charge leur avenir, en explorant des pistes fort diverses selon les cas (Kinahrejo palliant les manques de l'action publique pour accélérer sa relocalisation, Kalitengah Lor choisissant délibérément de conserver sa territorialité traditionnelle). Suffisamment ouvertes pour avoir laissé émerger des médiateurs et des forums hybrides, les communautés villageoises touchées ont un poids suffisant pour faire pression sur les autorités en cas de nécessité.

La relocalisation ouvre donc un entre-deux temporel et spatial qui est un champ pour des expériences dont sa réussite peut dépendre ; mais elle est aussi une table rase qui n'est pas sans risques. Des médiateurs (types 1 et 2 ) ouvrent ainsi deux pistes de réflexions pouvant déboucher sur de multiples possibles : la norme (norma) et la culture (budaya).

Mêlant coutume (adat) et droit positif (hukum), les normes intègrent des dimensions morales et juridiques. Se trouvent mises ici en jeu les notions de droits et de devoirs réciproques de chacun, dans une vision du monde $\left(k_{e j a w e n}{ }^{18}\right)$ où tout pouvoir a un fondement divin, toute règle un aspect sacré et tout évènement un sens cosmique. Des débats divisent ainsi les marga touchées (et plus généralement la société indonésienne) quant au statut des victimes (coupables de leur propre malheur ?) ou aux politiques de prévention (moraliser la société, ou faire confiance aux techniques scientifiques et/ou vernaculaires ?). Par-delà ces débats et les fractures idéologiques et/ou religieux qu'ils révèlent, la "théologie de la catastrophe " (Dupuy, 2005), semble rester populaire parmi toutes les générations. Face à cela, un médiateur de type 1 souligne - en privé et non en

\footnotetext{
${ }^{18}$ Vision holistique du monde, elle lie une vision hiérarchique et ritualisée de la société et de la vie, un fort attachement à la terre et un syncrétisme assimilé à un soufisme local et mêlant des apports islamiques, hindobouddhiques et animistes (Bing, à paraître).
} 
public - comme enjeu d'une relocalisation réussie d'éviter l' « appropriation bureaucratique de la souffrance » par les "cadres institutionnels» (Vásquez Lezama, 2009: 198): administrations, religions...

Des expressions culturelles qui se saisiraient de cette catastrophe afin de l'interpréter favoriseraient ainsi l'autonomie des villageois et la résilience. Des médiateurs (types 1 et 2) s'interrogent d'ailleurs quant à l'interdépendance entre transmission culturelle et cohésion des communautés. Or la transmission paraît assez solidement assurée : la pesantren al-Qodir fait du kejawen un fondement de son enseignement, Pentingsari assoit son développement touristique sur les pratiques artisanales et artistiques et Kinahrejo doit sa célébrité nationale et son attractivité touristique à sa centralité spirituelle. En revanche, peu posent la question de la création; et, sous cet angle, la culture javanaise (budaya Jawa) exprimée en javanais (bahasa Jawa) et le kejawen subissent la concurrence de la culture nationale islamomoderniste en indonésien (Raillon 2007) qui menace de les scléroser. II existe certainement des alternatives à la dualité transmission fidèle mais sclérosante relevant du javanais/création relevant du national ; mais cela reste à imaginer et à développer sur place.

Les élites des villages relocalisés (incarnées par les médiateurs) ont donc fait le choix d'une résilience non par un retour à la normale (cas de Kali Tengah Lor après 2010 ou de TurgoTritis après 1994) mais la recherche d'une nouvelle normalité. Cela oblige à repenser toute la territorialité des habitants, depuis l'échelle du micro-espace domestique jusqu'à celle du volcan dans son entier. Les communautés villageoises de Kinahrejo, Jambu et Kaliadem vivent ainsi la période post-catastrophe comme un temps d'incertitudes brutales. Considérer la situation sous l'angle géographique de l'entre-deux offre aux médiateurs et au forum mandataire de cet article une opportunité pour lier divers aspects (culture, économie, habitat quotidien, pendularité, cohésion sociale) de leur réflexion.

\section{Bibliographie}

AUGENDRE M. (2004), « Le risque naturel devenu symbiose ? Les volcans actifs d'Hokkaidô, Japon », Géomorphologie : relief, processus, environnement, vol. 10, n² 2, p.101-116.

BERLINER D. (2010), « Anthropologie et transmission », Terrain, $n^{\circ}$ 55, p. 4-19.

BING J.B. (2009), Savoirs locaux à propos des comportements animaux et stratégies populaires de prévention des risques, Mémoire de Master 2 sous la direction de F. Lavigne, Université Paris 1, $84 \mathrm{p}$.

BING J.B. (2014), « Métisser les savoirs pour négocier avec et sur un volcan. Javanité et forêt sur le Merapi », Revue de géographie alpine [en ligne], 9 p.

URL : http://rga.revues.org/2276 (30/05/2014)

CALLON M., LASCOUMES P., BARTHE Y. (2001), Agir dans un monde incertain, Paris, Seuil, $362 \mathrm{p}$.

DAUPHINÉ A. (2005), Risques et catastrophes. Observer, spatialiser, comprendre, gérer, Paris, A. Colin, $288 \mathrm{p}$.

DUPUY J.P. (2005), Petite métaphysique des tsunamis, Paris, Seuil, 107 p.

LAVIGNE F. (2001), " Le paradoxe d'une montagne tropicale volcanique : surpeuplement et risques naturels sur le volcan Merapi, Java, Indonésie ", in. BART F., MORIN S., SALOMON 
J.N. (dir.), Les Montagnes tropicales. Identités, mutations, développement, Pessac, CRET, pp. 119-131.

LAVIGNE F. et DE BELIZAL E. (2010), "Les effets géographiques des éruptions volcaniques », EchoGéo, Surlevif, 14 p.

URL : http://echogeo.revues.org/12226 (21/12/2013)

LAPIERRE N. (2004), Pensons ailleurs, Paris, Gallimard, $370 \mathrm{p}$.

LEVANG P. (1997), La terre d'en face : la transmigration en Indonésie, Paris, ORSTOM, 419 p.

MELCHIOR A. (2011), "Le Mérapi : la "Montagne de feu" au centre de l'île de Java, en Indonésie ", Lave, $\mathrm{n}^{\circ} 149$, p. 8-14.

MEI E.T.W., LAVIGNE F. et al. (2013), « Lessons learned from the 2010 evacuations at Merapi volcano ", Journal of Volcanology and Geothermal Research, $18 \mathrm{p}$.

URL : http://dx.doi.org/10.1016/j.jvolgeores.2013.03.010 (21/12/2013)

PÉGUY C.P. (2001), Espace, temps, complexité. Vers une métagéographie, Paris, Belin, 283 p.

RAILLON F. (2007), Indonésie. Les voies de la survie, Paris, Belin, 223 p.

RAFFESTIN C. (1986), "Écogenèse territoriale et territorialité », in. Auriac F., Brunet R. (dir.), Espaces, jeux et enjeux, Paris, Fayard, p. 173-185.

RAFFESTIN C. (1995), "Langue et territoire. Autour de la géographie culturelle ", in WALTY S. et WERLEN B. (éd.), Kulturen und Raum : theoretische Ansätze und empirische Kulturforschung in Indonesien : Festschrift für Professor Albert Leemann, Zurich, Rüegger, p. $87-104$.

TUAN Y.F. (2006) Espace et lieu. La perspective de l'expérience, Gollion, Infolio, 219 p.

VÁSQUEZ LEZAMA P. (2009), "De la tragédie collective à l'individuation du malheur. L'expérience de fin de légitimité de la condition de victime des sinistrés de la catastrophe de La Tragedia (1999) au Venezuela ", Anthropologie et sociétés, vol. 33, n³ 3, p. 193-210. 